\title{
Mediastinal Pancreatic Pseudocysts
}

\author{
Krzysztof Dąbkowski ${ }^{1}$, Andrzej Białek ${ }^{1}$, Maciej Kukla ${ }^{1}$, Janusz Wójcik ${ }^{2}$, Andrzej Smereczyński ${ }^{1}$, Katarzyna Kołaczyk ${ }^{3}$, \\ Tomasz Grodzki and Teresa Starzyńska ${ }^{2}$ \\ Departments of ${ }^{1}$ Gastroenterology, ${ }^{2}$ Thoracic Surgery and Transplantation, and ${ }^{3}$ Radiology, Pomeranian Medical University, Szczecin, Poland
}

Mediastinal pseudocysts are a rare complication of acute pancreatitis. Lack of uniform treatment standards makes the management of this condition a clinical challenge. We report the case of a 43-year-old patient who presented with a left pleural effusion. Pleural fluid revealed a high amylase concentration consistent with a pancreaticopleural fistula. Endoscopic retrograde cholangiopancreatography (ERCP) revealed a disruption of the pancreatic duct with free outflow of contrast medium into the thoracic cavity. A pancreatic stent was placed. The second day after the ERCP, the patient developed septic shock and was admitted to the intensive care unit. Computed tomography (CT) revealed mediastinal pseudocysts and bilateral pleural effusions. After bilateral drainage of the pleural cavities, the patient improved clinically, and a follow-up CT scan showed that the fluid collection and pseudocysts had resolved. We discuss the optimal strategies for diagnosing and treating patients with pancreatic thoracic pseudocysts and fistulas, as well as review the management of these conditions. Clin Endosc 2017;50:76-80

Key Words: Acute pancreatitis; Cholangiopancreatography, endoscopic retrograde; Mediastinal pseudocysts

\section{INTRODUCTION}

Pancreatic thoracic pseudocysts and pancreaticopleural fistulas are rare complications of acute and chronic pancreatitis. These conditions have symptoms that are often misleading. Moreover, no uniform diagnostic and treatment standards exist. We present a case of a patient with sepsis secondary to infected ascending mediastinal pancreatic pseudocysts, which are a rare complication of endoscopic retrograde cholangiopancreatography (ERCP). Thereafter, we present effective treatment methods and discuss optimal management strategies.

Received: May 25, 2016 Revised: August 30, 2016

Accepted: August 31, 2016

Correspondence: Krzysztof Dąbkowski

Department of Gastroenterology, Pomeranian Medical University, Uni Lubelskiej 1, 71-252 Szczecin, Poland

Tel: +48-503709056, Fax: +48-914253211, E-mail: dabkowskikrzysztof@wp.pl

(cc) This is an Open Access article distributed under the terms of the Creative Commons Attribution Non-Commercial License (http://creativecommons.org/ licenses/by-nc/3.0) which permits unrestricted non-commercial use, distribution, and reproduction in any medium, provided the original work is properly cited.

\section{CASE REPORT}

A 43-year-old man with bronchial asthma and three episodes of acute pancreatitis (AP, suspected etiology due to steroids) presented with exertional dyspnea approximately 1 month prior to admission. There was no evidence of AP on admission; the last episode had been 6 months before. The patient was in generally good health. Laboratory tests revealed mild anemia (hemoglobin $12.0 \mathrm{~g} / \mathrm{dL}$ ) and thrombocytosis (platelets $677 \times 10^{9} / \mathrm{L}$ ).

A chest radiograph showed fluid in the left pleura reaching the fifth rib in the mid-axillary line (Fig. 1). An abdominal computed tomography (CT) scan revealed pancreatic pseudocysts that communicated with the mediastinum. Thoracocentesis was performed. This resulted in $900 \mathrm{~mL}$ of a clear, yellowish fluid with a high amylase (14,449 IU/L) and protein $(3.5 \mathrm{~g} / \mathrm{dL})$ content. The diagnosis of a pancreaticopleural fistula was made. A two-stage approach was chosen to manage the fistula: first, pancreatic ductal stenting of the leakage site via ERCP, followed by transgastric or transduodenal endoscopic pseudocyst drainage.

ERCP revealed leakage of contrast medium into the thoracic cavity in the proximal portion of the pancreatic tail (Fig. 2). 


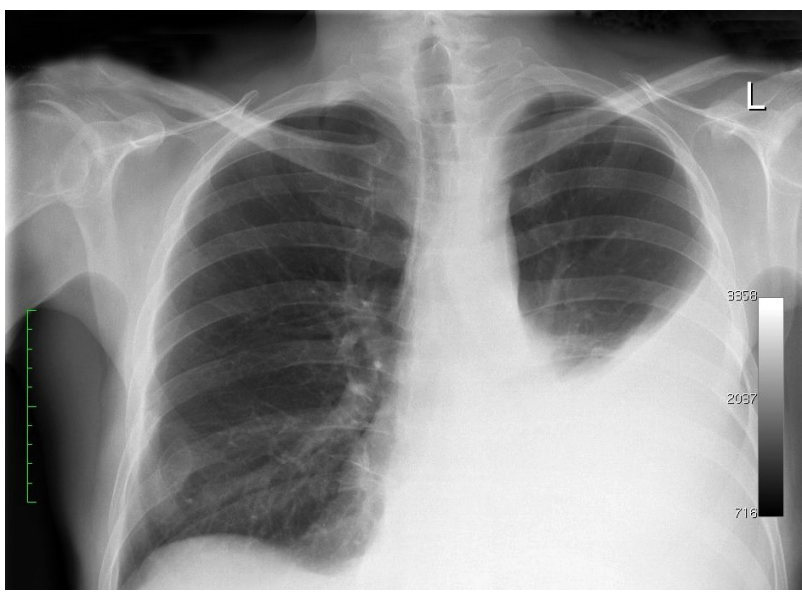

Fig. 1. Chest radiograph demonstrating fluid in the left pleural cavity.

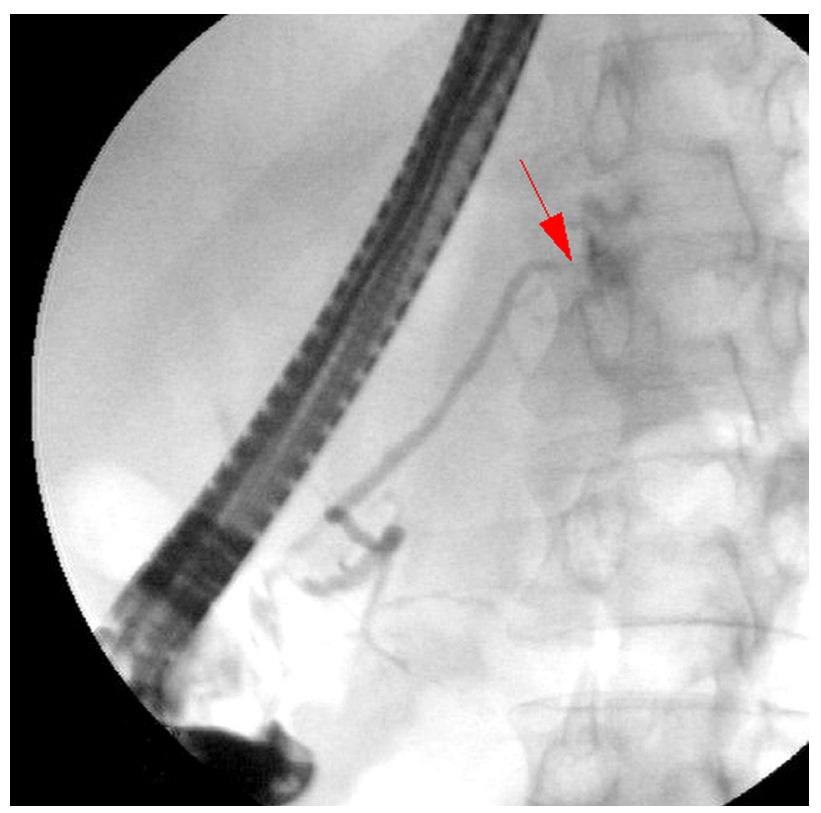

Fig. 2. Endoscopic retrograde cholangiopancreatography showing free outflow of the contrast medium from the proximal portion of the pancreatic tail to the mediastinum (the arrow points to the "leakage").

A stent was placed in the pancreatic duct (Fig. 3). Initially, the patient started to recover after the procedure. However, on the second day after ERCP, he deteriorated rapidly and developed clinical signs of septic shock. Laboratory tests revealed elevated inflammatory markers, including a procalcitonin level of $10 \mathrm{ng} / \mathrm{mL}$, and elevated serum (760 IU/L) and urinary amylase $(4,416 \mathrm{IU} / \mathrm{L})$.

The patient improved clinically after fluid resuscitation and the administration of broad-spectrum antibiotics (meropenem). However, he continued to require intravenous amiodarone for persistent tachycardia. Continuous intravenous somatostatin and total parenteral nutrition were also administered. He developed dyspnea, chest pain, and vomiting, but further

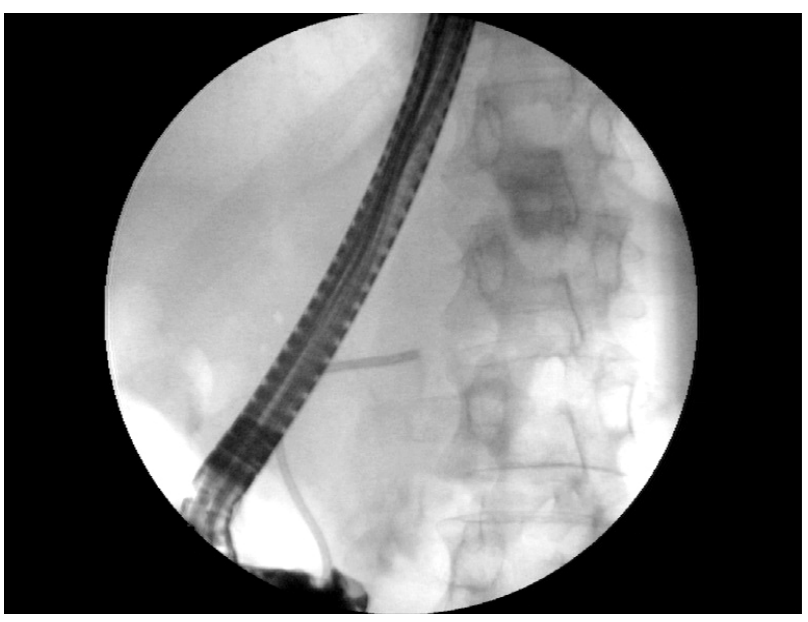

Fig. 3. Endoscopic retrograde cholangiopancreatography showing the location of the stent in the pancreatic duct. The stent is placed distally to the fistula, as close as possible to the leakage point.
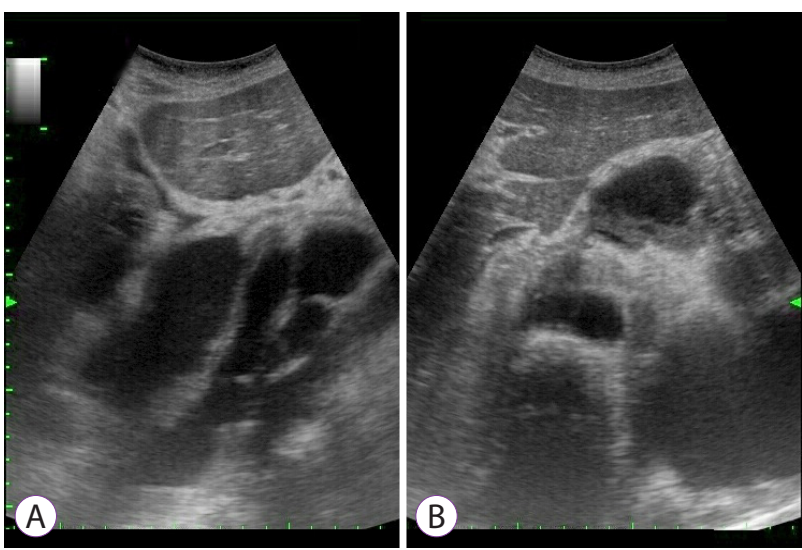

Fig. 4. (A, B) Abdominal ultrasound depicting pseudocysts in the posterior mediastinum.

evaluation did not reveal any signs of myocardial infarction. An abdominal ultrasound (Fig. 4) and chest CT scan revealed posterior mediastinal pseudocysts extending from the pancreas through the diaphragmatic hiatus to the mediastinum and neck, with compression of the surrounding tissues and fluid in both pleural cavities (Fig. 5). Bilateral drainage of the pleural cavities was performed in the thoracic surgery ward. This comprised 2 weeks of active and 1 week of ambulatory passive drainage. Thereafter, chest radiography revealed regression of the pleural fluid (Fig. 6). The patient's inflammatory markers improved, and he was discharged home after 3 weeks of staying in hospital in a satisfactory physical condition. A follow-up CT scan performed at 12 months showed that the pseudocysts and pleural fluid had resolved (Fig. 7). He remained symptom-free throughout the 26-month follow-up period. 


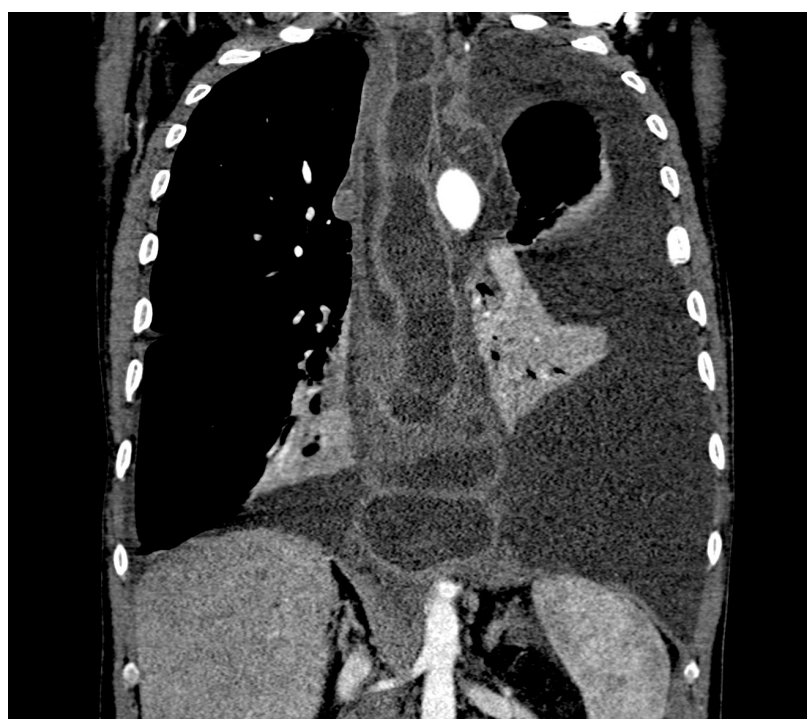

Fig. 5. Computed tomography scan of the chest showing mediastinal pseudocysts and pleural fluid.

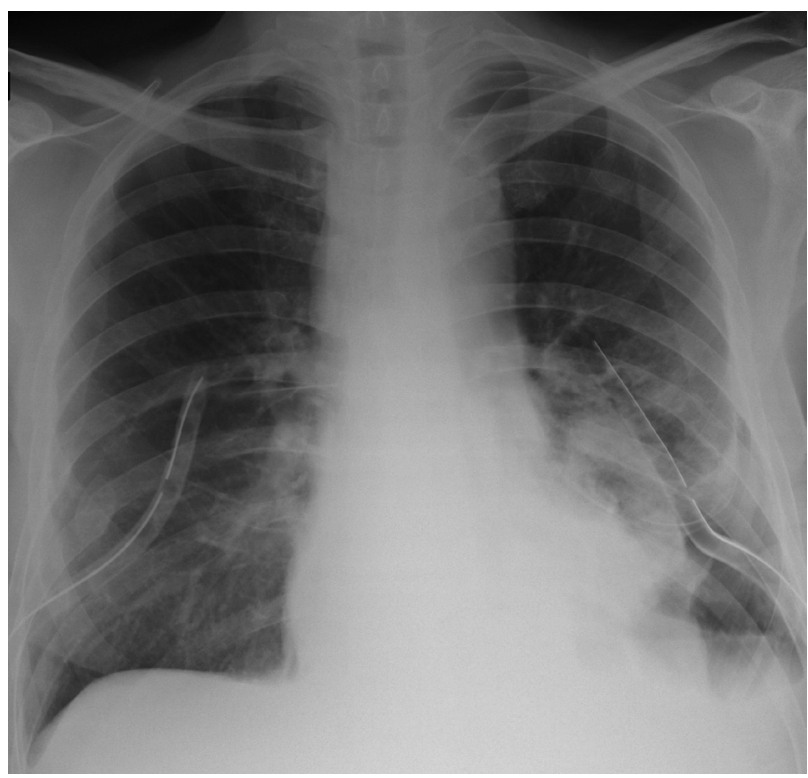

Fig. 6. Chest radiograph showing fluid resolution after pleural fluid drainage.

\section{DISCUSSION}

Thoracic pancreatic pseudocysts and pancreaticopleural fistulas are rare complications of acute and chronic pancreatitis, as well as trauma. ${ }^{1}$ They are triggered by the disruption of the pancreatic duct, which leads to pancreatic fluid penetrating through the diaphragmatic orifices (usually the aortic or esophageal hiatus) into the mediastinum. There, it can transform into a pleural fistula or a mediastinal pseudocyst. ${ }^{1,2}$ To the best of our knowledge, there have been fewer than 100 cases of mediastinal pancreatic cyst expansion reported in the literature. ${ }^{3}$ While the precise incidence is unknown,

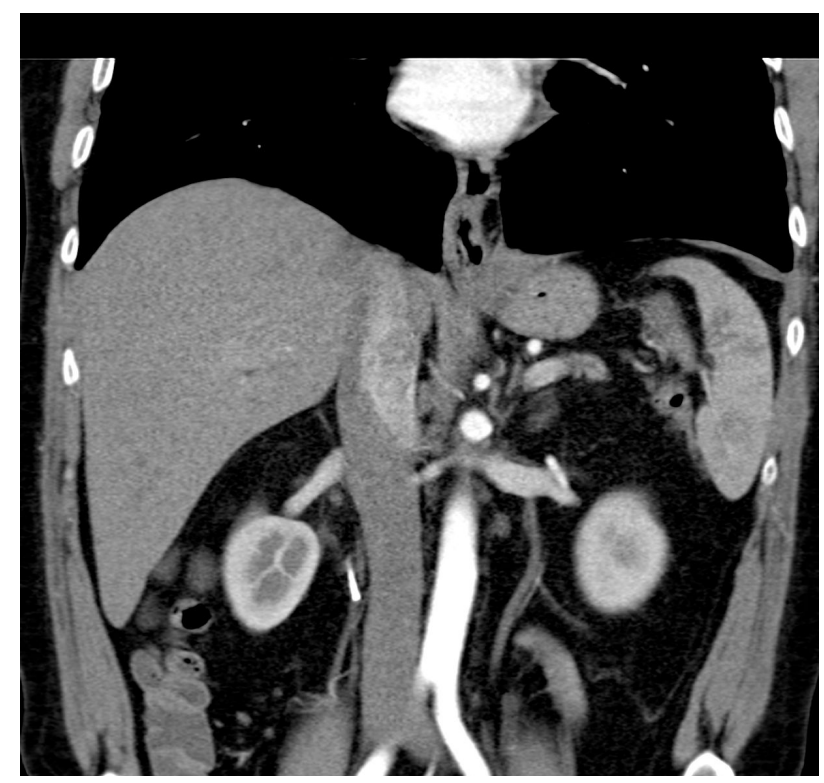

Fig. 7. Resolution of pseudocysts on the 12-month follow-up computed tomography scan.

the frequency of pancreaticopleural fistulas in patients with pancreatitis is estimated to be $0.4 \%$ to $4.5 \% .{ }^{4.5}$ Approximately $80 \%$ of cases of pancreaticopleural fistulas are associated with mediastinal pseudocysts. ${ }^{6}$

The symptoms are often misleading, and typically result from significant pleural effusions or the compression of mediastinal structures. Pulmonary symptoms such as dyspnea, cough, chest pain, or dysphagia, can be present, as well as life-threatening complications like hemoptysis, cardiogenic shock, and acute respiratory failure. ${ }^{7}$ Abdominal symptoms such as pain are rare. ${ }^{5}$

The diagnosis is typically established by multiple radiologic tests, including ultrasound, CT, magnetic resonance cholangiopancreatography (MRCP), ERCP, and pleural fluid analysis. The pleural fluid is typically characterized by a high amylase and albumin content. ${ }^{5}$ The mean amylase level in the pleural fluid of patients with pancreaticopleural fistulas may exceed 10,000 IU/L. ${ }^{8}$ ERCP and MRCP are helpful in establishing the fistulous communication between the pancreatic duct and pleura. ERCP leads to the diagnosis in $80 \%$ of cases, demonstrates the fistulous tract in 59\%, and also allows for pancreatic duct stenting. ${ }^{9}$

There are many strategies to treat thoracic pancreatic pseudocysts. These depend on the anatomy, pseudocyst size, and presence and severity of symptoms. ${ }^{7}$ Conservative therapy with somatostatin analogs, total parenteral nutrition, and/or bromhexine hydrochloride ${ }^{5,10,11}$ can be successful for asymptomatic patients with small cysts. ${ }^{10}$ Large or symptomatic pseudocysts require invasive therapies such as surgery (distal pancreatectomy, pancreatic head resection, cystojejunostomy, cystogas- 
trostomy, or Puestow procedure) or drainage. ${ }^{12,13}$ Drainage procedures can be performed percutaneously under CT or ultrasound guidance; ${ }^{7,14}$ alternatively, they may be performed endoscopically via the gastrointestinal tract wall through the transesophaegal, transgastric, or transpapillary approach. ${ }^{15,16}$ The advantage of endoscopic treatment is the lower risk of complications compared to percutaneous drainage. ${ }^{7}$ However, due to the small number of pancreatic pseudocysts described in the literature, there has not been any studies comparing the efficacy and safety of different types of endoscopic drainage.

Ajmera and Judge ${ }^{7}$ presented an algorithm for the treatment of mediastinal pancreatic pseudocysts. They proposed that all unstable patients with life-threatening complications should be treated with open surgery, while the management of stable patients should depend on the size of the pseudocyst and the presence of symptoms. Symptomatic patients or those with large cysts should be treated with drainage. Endoscopic drainage is preferred, especially if the procedure is performed in a tertiary referral center and the anatomy is favorable, which means the cyst is located near the gastric wall or is communicating with the pancreatic duct. Asymptomatic patients should be treated conservatively and followed up.? Pseudocysts complicated by infection, obstruction, rupture, or hemorrhage should be treated surgically. ${ }^{\text {? }}$

There have also been attempts to introduce thoracosurgical treatment methods for thoracic pancreatic pseudocysts. For example, Chang and $\mathrm{Chen}{ }^{17}$ has reported the successful use of video-assisted thoracoscopic surgery with bilateral drainage of the pleural cavity in treating ascending mediastinitis arising from pancreatic pseudocysts.

In cases of pancreaticopleural fistulas, suppression of exocrine pancreatic function with octreotide and ERCP with fistula stenting is optimal, and has a low morbidity and mortality rate. ${ }^{18}$ Surgical treatment is more effective with a success rate of $90 \%$; however, it is associated with a higher mortality rate, and therefore remains a second-line option. ${ }^{18}$ Wronski et al. ${ }^{4}$ proposed an algorithm to treat pancreaticopleural fistulas: all patients should be assessed with MRCP prior to treatment, and the treatment method should depend on the pancreatic ductal abnormality. Patients with non- or a mildly dilated pancreatic duct without stenosis should have a trial of conservative treatment for 2 to 3 weeks. If ductal strictures and ductal disruption in the pancreatic head or body are present, ERCP should be used as first-line treatment. If complete ductal obstruction or strictures and fistulas in the pancreatic tail are present, surgery should be considered the first-line treatment instead. ${ }^{4}$

In this present case, we aimed to improve the patient's clinical status and resolve both the pleural fluid and the pseudocysts. As previously described, a two-stage therapeutic strategy was employed: first, the location of the leak was stented by ERCP; second, the abdominal portion of the pseudocyst was drained through the gastrointestinal tract via the transgastric or transesophageal approach. However, in retrospect, the patient could have been more optimally assessed with MRCP and/or chest CT before ERCP took place. The patient's post-ERCP course was complicated by life-threatening sepsis, which was initially misdiagnosed as AP secondary to the ERCP. However, the patient had dominating "chest" symptoms, including dyspnea, chest pain, and recurrent paroxysmal tachycardia. In addition, the ultrasound and CT scan showed polycyclic pseudocysts extending from the pancreas through the mediastinum to the neck beyond the scanning border with compression of surrounding tissues. This led us to the diagnosis of ascending post-ERCP mediastinal pseudocyst inflammation. Due to the patient's unstable condition and the risk of life-threatening complications, he was transferred to the thoracic surgery ward, where effective bilateral pleural drainage was performed. Our treatment approach was based on the findings of the pleural fluid communicating with the mediastinal pseudocysts and the pancreatic duct. We also believed that a pancreatic duct stent would prevent further pseudocyst expansion.

The patient's clinical status eventually improved, his symptoms resolved, and he was discharged home on day 21 after admission. His hospital stay consisted of 1 week in the department of gastroenterology, and 2 weeks in the department of thoracic surgery. A 12-month follow-up abdominal CT scan showed that the pseudocysts had resolved. To the best of our knowledge, this case of complete pseudocyst resolution after bilateral pleural drainage, preceded by stenting of the pancreatic duct, has not been described in the literature.

In summary, pancreatic duct stenting followed by bilateral pleural fluid drainage can be an effective treatment of thoracic pancreatic pseudocysts in the context of a coexisting pancreaticopleural fistula. We believe that every patient with thoracic pancreatic pseudocysts and/or pancreaticopleural fistulas should be carefully assessed by a multidisciplinary team-including gastroenterologists, surgeons, thoracic surgeons, and radiologists - to determine the optimal management strategy.

Conflicts of Interest

The authors have no financial conflicts of interest.

\section{REFERENCES}

1. Gupta R, Munoz JC, Garg P, Masri G, Nahman NS Jr, Lambiase LR. Mediastinal pancreatic pseudocyst: a case report and review of the literature. MedGenMed 2007;9:8.

2. Dyrla P, Gil J, Wojtuń S, Florek M, Kasińska E, Zoń R. Pancreatic 
pseudocyst in the mediastinum. Pol Arch Med Wewn 2014;124:270-271.

3. Moorthy N, Raveesha A, Prabhakar K. Pancreaticopleural fistula and mediastinal pseudocyst: an unusual presentation of acute pancreatitis. Ann Thorac Med 2007;2:122-123.

4. Wronski M, Slodkowski M, Cebulski W, Moronczyk D, Krasnodebski IW. Optimizing management of pancreaticopleural fistulas. World J Gastroenterol 2011;17:4696-4703.

5. Dhebri AR, Ferran N. Nonsurgical management of pancreaticopleural fistula. JOP 2005;6:152-161.

6. Altasan T, Aljehani Y, Almalki A, Algamdi S, Talag A, Alkattan K. Pancreaticopleural fistula: an overlooked entity. Asian Cardiovasc Thorac Ann 2014;22:98-101.

7. Ajmera AV, Judge TA. Mediastinal extension of pancreatic pseudocyst: a case with review of topic and management guidelines. Am J Ther 2012;19:e152-e156.

8. Tay CM, Chang SK. Diagnosis and management of pancreaticopleural fistula. Singapore Med J 2013;54:190-194.

9. Safadi BY, Marks JM. Pancreatic-pleural fistula: the role of ERCP in diagnosis and treatment. Gastrointest Endosc 2000;51:213-215.

10. Suga $\mathrm{H}$, Tsuruta O, Okabe $\mathrm{Y}$, et al. A case of mediastinal pancreatic pseudocyst successfully treated with somatostatin analogue. Kurume Med J 2005;52:161-164
11. Tsujimoto T, Takano M, Tsuruzono T, et al. Mediastinal pancreatic pseudocyst caused by obstruction of the pancreatic duct was eliminated by bromhexine hydrochloride. Intern Med 2004;43:1034-1038.

12. Ali T, Srinivasan N, Le V, Chimpiri AR, Tierney WM. Pancreaticopleural fistula. Pancreas 2009;38:e26-e31.

13. Belágyi T, Issekutz A, Paukovics A, Kecskés G, Oláh A. Successful treatment of mediastinal pancreatic pseudocyst by pancreatic head resection. JOP 2008;9:346-349.

14. Takeuchi Y, Okabe H, Myojo S, Fujimoto S. CT-guided drainage of a mediastinal pancreatic pseudocyst with a transhepatic transdiaphragmatic approach. Hepatogastroenterology 2002;49:271-272.

15. Săftoiu A, Ciurea T, Dumitrescu D, Stoica Z. Endoscopic ultrasound-guided transesophageal drainage of a mediastinal pancreatic pseudocyst. Endoscopy 2006;38:538-539.

16. Topa L, László F, Sahin P, Pozsár J. Endoscopic transgastric drainage of a pancreatic pseudocyst with mediastinal and cervical extensions. Gastrointest Endosc 2006;64:460-463.

17. Chang YC, Chen CW. Thoracoscopic drainage of ascending mediastinitis arising from pancreatic pseudocyst. Interact Cardiovasc Thorac Surg 2009;9:144-145.

18. Machado NO. Pancreaticopleural fistula: revisited. Diagn Ther Endosc 2012;2012:815476. 